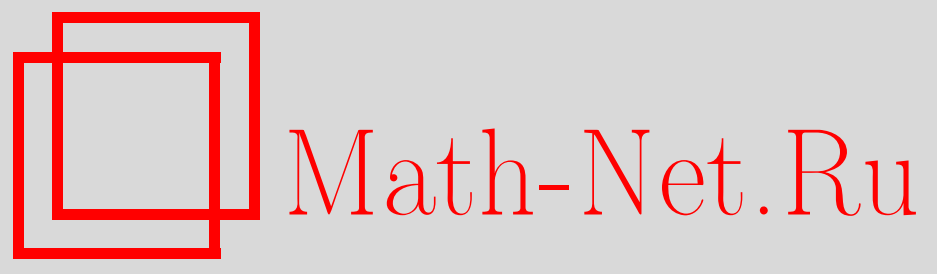

Е. В. Антропова, А. А. Брызгалов, Ф. И. Карманов, Уровни энергии и собственные волновые функции электронов систем квантовых колец в магнитном поле, Вестн. Сам. гос. техн. ун-та. Сер. Физ.мат. науки, 2013, выпуск 1(), 326-333

DOI: https://doi.org/10.14498/vsgtu1171

Использование Общероссийского математического портала Math-Net.Ru подразумевает, что вы прочитали и согласны с пользовательским соглашением

http: //www . mathnet.ru/rus/agreement

Параметры загрузки:

IP : 3.81 .55 .215

26 апреля 2023 г., 13:48:08




Вестн. Сам. гос. техн. ун-та. Сер. Физ.-мат. науки. 2013. № 1 (30). С. $326-333$

УДК 51:530.145

\title{
УРОВНИ ЭНЕРГИИ И СОБСТВЕННЫЕ ВОЛНОВЫЕ ФУНКЦИИ ЭЛЕКТРОНОВ СИСТЕМ КВАНТОВЫХ КОЛЕЦ В МАГНИТНОМ ПОЛЕ
}

\author{
Е. В. Антропова, А.А. Брызгалов, Ф.И. Карманов \\ Обнинский институт атомной энергетики - филиал НИЯУ МИФИ \\ (национальный исследовательский ядерный университет «МИФИ»), \\ Россия, 249040, Обнинск, ул. Студгородок, 1. \\ E-mails: antrolen@yandex.ru, sandro185@mail.ru, fikarm@yandex.ru
}

\begin{abstract}
Предложен вид потенциала, создаваемого квантовым колъцом, для которого можно получить аналитическое решение стационарного уравнения Шрёдингера. Найдено решение соответствующей задачи на собственные значения в терминах функций Хойна. Получено выражение для энергетических уровней невзаимодействующих электронов в квантовом колъще в присутствии магнитного поля. Рассмотрены возможные сферы применения данной модели.
\end{abstract}

Ключевые слова: квантовое кольцо, магнитное поле, квазиточнорешаемые модели квантовой механики.

Введение. В связи с развитием электроники и нанотехнологий возрос интерес к моделированию квантовых низкоразмерных систем с целью их изучения и дальнейшего использования. Поскольку создаваемые на практике наноструктуры обладают очень широким многообразием форм и размеров, определить вид потенциала, ограничивающего движение в них заряженных частиц (электронов), чрезвычайно трудно, поэтому нужны математические модели, позволяющие получать точные (или почти точные) решения. Тогда, сравнивая результаты эксперимента и модельного расчета, можно получить значения параметров модельного потенциала для конкретных структур и применять их в дальнейшем.

K числу почти точно или квазиточнорешаемых задач относятся такие задачи, в которых в явном замкнутом виде удается получить не весь спектр исследуемой системы, а только его некоторую часть [1]. Часто это выражается в том, что для исследуемого спектра системы получается некоторое рекуррентное соотношение, позволяющее вычислить требуемый участок точно или асимптотически точно, решая численно некоторые трансцендентные уравнения. Точно решаемые модели в квантовой физике важны сами по себе как модели реальных систем. Эти модели позволяют провести проверку точности численных процедур решения соответствующих дифференциальных уравнений и качества используемых приближений. Кроме того, они важны для оценки вкладов и поправок, вносимых в процессе аналитических расчетов методами теории возмущений. В работах $[2,3]$ предложены точно решаемые модели, которые могут быть использованы для изучения свойств квантовых колец и квантовых точек, находящихся в магнитном поле.

В некоторых подходах к решению нестационарного уравнения Шрёдингера требуется базис из собственных функций, являющихся решениями стаци-

Елена Bикторовна Антропова, магистрант, каф. общей и специльной физики. Александр Анатольевич Брызгалов, аспирант, каф. общей и специльной физики. Фёдор Иванович Карманов (к.ф.-м.н., доц.), доцент, каф. общей и специльной физики. 
онарной задачи [4]. Однако существует не так много типов потенциалов, для которых можно получить аналитическое решение. В данной работе предлагается следующая параметризация потенциала квантового кольца:

$$
V(r)=\frac{a_{-2}}{r^{2}}+\frac{a_{-1}}{r}+a_{1} r+a_{2} r^{2}+V_{0}
$$

где $a_{-2}, a_{-1}, a_{1}, a_{2}, V_{0}$ - некоторые константы.

1. Постановка задачи. Рассматривается модель невзаимодействующих электронов, движущихся в аксиально-симметричном квантовом кольце, характеризуемом внутренним и внешним радиусами $R_{1}$ и $R_{2}$, в присутствии постоянного магнитного поля $\vec{B}$, перпендикулярного плоскости кольца (рис. 1$)$. Задана цилиндрическая система координат с началом отсчёта в центре кольца. Считается, что высота кольца достаточно мала, чтобы кольцо можно было считать плоским и не учитывать зависимость поля от переменной $z$. Ставится задача на собственные значения для стационарного уравнения Шрёдингера

$$
\widehat{H} \psi(r, \varphi)=E \psi(r, \varphi)
$$

с гамильтонианом следующего вида:

$$
\widehat{H}=\frac{\hbar^{2}}{2 \mu}\left(-\frac{1}{r} \frac{\partial}{\partial r}\left(r \frac{\partial}{\partial r}\right)-\frac{1}{r^{2}} \frac{\partial^{2}}{\partial^{2} \varphi}-i \frac{q B}{\hbar} \frac{\partial}{\partial \varphi}+\frac{q^{2} B^{2}}{4 \hbar^{2}} r^{2}\right)+V(r)
$$

где $\hbar$ - постоянная Планка, $\mu$-эффективная масса электрона, $B$ - индукция магнитного поля, $V(r)$ - модельный потенциал. $\Psi$-функция должна удовлетворять граничным условиям для радиальной составляющей $\psi(0, \varphi)=$ $=\psi(\infty, \varphi)=0$, условию периодичности $\psi(r, \varphi)=\psi(r, \varphi+2 \pi)$ и условию



Рис. 1. Модель квантового кольца 
нормировки

$$
\langle\psi(r, \varphi) \mid \psi(r, \varphi)\rangle=\int_{0}^{2 \pi} \int_{0}^{\infty} \psi^{*}(r, \varphi) \psi(r, \varphi) r d r d \varphi=1 .
$$

2. Переход к уравнению Хойна. Волновая функция ищется в виде произведения функции, зависящей от $r$, и фазового множителя, зависящего от угла $\varphi$ :

$$
\psi(r, \varphi)=e^{i m \varphi} f(r) / \sqrt{2 \pi}
$$

где $m$-магнитное квантовое число. При этом $f(r)$ оказывается нормированной на единицу. После подстановки (3) в (2) получается уравнение для радиальной части волновой функции:

$$
r^{2} \frac{d^{2}}{d^{2} r} f(r)+r \frac{d}{d r} f(r)-\left(C_{0}^{2}+C_{1} r+C_{2} r^{2}+C_{3} r^{3}+C_{4}^{4} r^{4}\right) f(r)=0
$$

где введены следующие обозначения:

$$
\begin{gathered}
C_{0}=\left(m^{2}+\frac{2 \mu a_{-2}}{\hbar^{2}}\right), \quad C_{1}=\frac{2 \mu a_{-1}}{\hbar^{2}}, \quad C_{2}=\frac{q B m}{\hbar}-\frac{2 \mu\left(E-V_{0}\right)}{\hbar^{2}}, \\
C_{3}=\frac{2 \mu a_{1}}{\hbar^{2}}, \quad C_{4}=\left(\frac{q^{2} B^{2}}{4 \hbar^{2}}+\frac{2 \mu V_{0}}{\hbar^{2}}\right) .
\end{gathered}
$$

Подставив в (4) решение в виде

$$
f(r)=\exp \left(-\frac{r\left(C_{4}^{4} r+C_{3}\right)}{2 C_{4}^{2}}\right) r^{C_{0}} w(r)
$$

получим дифференциальное уравнение второго порядка относительно $w(r)$ :

$$
\begin{aligned}
r \frac{d^{2}}{d^{2} r} w(r)+ & \left(1+2 C_{0}-\frac{C_{3}}{C_{4}^{2}} r-2 C_{4}^{2} r^{2}\right) \frac{d}{d r} w(r)+ \\
& +\left(\left(\frac{C_{3}^{2}}{4 C_{4}^{4}}-2 C_{4}^{2}\left(C_{0}+1\right)-C_{2}\right) r-\frac{C_{3}\left(2 C_{0}+1\right)}{2 C_{4}^{2}}-C_{1}\right) w(r)=0 .
\end{aligned}
$$

Перейдём от переменной $r$ к $x=C_{4} r$ и набора констант $C_{0}, C_{1}, C_{2}, C_{3}, C_{4}$ к $\alpha, \beta, \gamma, \delta$ так, что

$$
\alpha=2 C_{0}, \quad \beta=\frac{C_{3}}{C_{4}^{3}}, \quad \gamma=\frac{C_{3}^{2}-4 C_{2} C_{4}^{4}}{4 C_{4}^{6}}, \quad \delta=\frac{2 C_{1}}{C_{4}},
$$

в результате получится биконфлюэнтное уравнение Хойна (в литературе также упоминается как уравнение Гойна [5]) в канонической форме:

$$
\begin{aligned}
x \frac{d^{2}}{d^{2} x} w(x)+(1+\alpha-\beta x & \left.-2 x^{2}\right) \frac{d}{d x} w(x)+ \\
& +\left((\gamma-\alpha-2) x-\frac{1}{2}(\delta+\beta(1+\alpha))\right) w(x)=0 .
\end{aligned}
$$


Его общее решение выражается через биконфлюэнтные функции Хойна $\operatorname{HB}(\alpha, \beta, \gamma, \delta, x)$ :

$$
w(x)=D_{1} \cdot \operatorname{HB}(\alpha, \beta, \gamma, \delta, x)+D_{2} \cdot r^{-\alpha} \operatorname{HB}(-\alpha, \beta, \gamma, \delta, x) .
$$

3. Граничное условие в нуле. Подставим общее решение (8) в выражение для радиальной части волновой функции с учётом значений констант (6):

$$
\begin{aligned}
f(r)=D_{1} \cdot \exp \left(-\frac{r\left(C_{4}^{4} r+C_{3}\right)}{2 C_{4}^{2}}\right) r^{C_{0}} \mathrm{HB}\left(\alpha, \beta, \gamma, \delta, C_{4} r\right)+ \\
\quad+D_{2} \cdot \exp \left(-\frac{r\left(C_{4}^{4} r+C_{3}\right)}{2 C_{4}^{2}}\right) r^{-C_{0}} \mathrm{HB}\left(-\alpha, \beta, \gamma, \delta, C_{4} r\right) .
\end{aligned}
$$

При $C_{0}>0$ первая компонента общего решения ограничена в нуле, а вторая нет, поэтому следует положить $D_{2}=0$. Константа $D_{1}$ находится из условия нормировки:

$$
D_{1}=\left(\int_{0}^{\infty} r^{2 C_{0}+1} \operatorname{HB}\left(2 C_{0}, \frac{C_{3}}{C_{4}^{3}}, \frac{C_{3}^{2}-4 C_{2} C_{4}^{4}}{4 C_{4}^{6}}, \frac{2 C_{1}}{C_{4}}, C_{4} r\right)^{2} d r\right)^{-1 / 2} .
$$

4. Граничное условие на бесконечности. Для того чтобы волновая функция обращалась в ноль на бесконечности, ограниченное в нуле частное решение уравнения Хойна (7) должно представлять собой полином. Решения такого вида существуют, если параметры функции $\mathrm{HB}(\alpha, \beta, \gamma, \delta, x)$ удовлетворяют двум соотношениям [6]:

$$
\gamma=\alpha+2+2 n \quad(n=0,1,2, \ldots)
$$

и

$$
A_{k+1}(\theta)=0
$$

где $k \geqslant 0$ - степень полинома,

$$
\theta=\frac{1}{2}(\delta+\beta(\alpha+1))
$$

Коэффициенты $A_{k}$ определяются следующими рекуррентными соотношениями:

$$
\begin{aligned}
A_{0} & =1, \\
A_{1} & =\theta, \\
A_{k+2} & =((k+1) \beta+\theta) A_{k+1}-(\gamma-2-\alpha-2 k)(k+1)(k+1+\alpha) A_{k} .
\end{aligned}
$$

Следует отметить, что условие (10) при заданных параметрах потенциала может выполняться не для всех подряд идущих $n$.

5. Выражения для энергий и волновых функций. Подставим (6) в (9) и выразим $C_{2}$ через остальные переменные:

$$
C_{2}=\frac{C_{3}^{2}}{4 C_{4}^{4}}-2\left(C_{0}+1+n\right) C_{4}^{2} .
$$


Теперь выразим из (5) $E$ через $C_{2}$ :

$$
E=V_{0}+\frac{q B m \hbar-\hbar^{2} C_{2}}{2 \mu} .
$$

Окончательно получим выражение для уровней энергии, подставив в (14) соотношения (5) для $C_{0}, C_{3}, C_{4}$, а также (13):

$$
\begin{aligned}
E=V_{0}+\frac{q B m \hbar}{2 \mu}- & \frac{2 a_{1}^{2} \mu}{q^{2} B^{2}+8 \mu a_{0}}+ \\
& \quad+\frac{\hbar}{\mu}\left(\frac{q^{2} B^{2}}{4}+2 \mu a_{0}\right)^{1 / 2}\left(n+1+\left(m^{2}+\frac{2 \mu a_{-2}}{\hbar^{2}}\right)^{1 / 2}\right) .
\end{aligned}
$$

Пусть $\left\{n_{1}, n_{2}, n_{3}, \ldots\right\}$ - последовательность таких значений $n$, для которых имеет место равенство (10), $N$ - номер энергетического уровня или главное квантовое число, тогда соответствующие ему энергия и волновая функция будут

$$
\begin{aligned}
& E_{N, m}=V_{0}+\frac{q B m \hbar}{2 \mu}-\frac{2 a_{1}^{2} \mu}{q^{2} B^{2}+8 \mu a_{0}}+ \\
& +\frac{\hbar}{\mu}\left(\frac{q^{2} B^{2}}{4}+2 \mu a_{0}\right)^{1 / 2}\left(n_{N}+1+\left(m^{2}+\frac{2 \mu a_{-2}}{\hbar^{2}}\right)^{1 / 2}\right) \\
& \begin{aligned}
\psi_{N}(r, \varphi, E)=\frac{D_{N}}{\sqrt{2 \pi}} \cdot \exp \left(-\frac{r\left(C_{4}^{4} r+C_{3}\right)}{2 C_{4}^{2}}\right) \cdot r^{C_{0}} \times \\
\quad \times \operatorname{HB}\left(2 C_{0}, \frac{C_{3}}{C_{4}^{3}}, 2 n_{N}+2+2 C_{0}, \frac{2 C_{1}}{C_{4}}, C_{4} r\right)
\end{aligned}
\end{aligned}
$$

где

$$
D_{N}=\left(\int_{0}^{\infty} r^{2 C_{0}+1} \mathrm{HB}\left(2 C_{0}, \frac{C_{3}}{C_{4}^{3}}, 2 n_{N}+2+2 C_{0}, \frac{2 C_{1}}{C_{4}}, C_{4} r\right)^{2} d r\right)^{-1 / 2}
$$

Данная система собственных функций является полной в силу того, что уравнение (7) заменой переменных

$$
w=x^{-\frac{1+\alpha}{2}} \cdot e^{\frac{\beta x+x^{2}}{2}} v(x)
$$

сводится к уравнению Шрёдингера вида

$$
\frac{d^{2}}{d^{2} x} v(x)+\left(-x^{2}+\beta x+\gamma-\frac{\beta^{2}}{4}-\frac{\delta}{2 x}+\frac{1-\alpha^{2}}{4 x^{2}}\right) \cdot v(x),
$$

которое вместе с краевыми условиями $v(0)=v(\infty)=0$ представляет собой частный случай задачи Штурма-Лиувилля с $k(x)=1, \rho(x)=1$ [7]. Для этой задачи справедливы результаты осциляционной теоремы, позволяющие 
утверждать, что задача имеет счётное число собственных значений и собственных функций, поэтому найденная система собственных функций может использоваться в качестве базиса.

6. Аналитическое решение задачи для потенциала с $a_{-1}=a_{1}=0$. В статье [2] рассматривается модель потенциала, в котором нет слагаемых типа $r$ и $1 / r$, что соответствует $a_{-1}=a_{1}=0$ в выражении (1). Для этого потенциала авторами этой работы были получены волновые функции и спектр энергий в терминах вырожденных гипергеометрических функций. Если подставить $a_{-1}=a_{1}=0$ в $(5)$ и $(6)$, то

$$
C_{1}=C_{3}=0, \quad \beta=\delta=0,
$$

а в соответствии с (11) и $\theta=0$, поэтому рекуррентные соотношения (12) запишутся как

$$
A_{k+2}=(2 k+2+\alpha-\gamma)(k+1)(k+1+\alpha) A_{k} \quad(k \geqslant 0) .
$$

Отсюда следует, что $n$ может принимать только чётные значения: $n=2 \nu, \nu=$ $=0,1,2, \ldots$, где $\nu$ обозначает номер энергетического уровня. С учётом этого выражение для энергий частицы приобретёт следующий вид:

$$
E_{\nu, m}=a_{0}+\frac{q B m \hbar}{2 \mu}+\frac{\hbar}{\mu}\left(\frac{q^{2} B^{2}}{4}+2 \mu a_{0}\right)^{1 / 2}\left(2 \nu+1+\left(m^{2}+\frac{2 \mu a_{-2}}{\hbar^{2}}\right)^{1 / 2}\right)
$$

а соответствующие волновые функции в терминах функций Хойна записываются как

$$
\psi_{\nu, m}=\frac{D_{\nu}}{\sqrt{2 \pi}} \cdot e^{-\frac{C_{4}^{2} r^{2}}{2}+i m \varphi} \cdot r^{C_{0}} \operatorname{HB}\left(2 C_{0}, 0,2\left(C_{0}+1+2 \nu\right), 0, C_{4} r\right),
$$

где

$$
D_{\nu}=\left(\int_{0}^{\infty} r^{2 C_{0}+1} \operatorname{HB}\left(2 C_{0}, 0,4 \nu+2+2 C_{0}, 0, C_{4} r\right)^{2} d r\right)^{-1 / 2}
$$



Рис. 2. Энергии электрона в зависимости от индукции магнитного поля и магнитного квантового числа: $1-B=0,0$ Тл; $2-B=0,2$ Тл; $3-B=0,5$ Тл; $4-B=1,0$ Тл 
В работе [2] были использованы следующие параметры потенциала: $a_{-2}=$ $=9,1022 \cdot 10^{6} \mathrm{м \ni B} \cdot \mathrm{Hм}^{2}, a_{2}=2,222 \cdot 10^{-5}$ мэВ $\cdot \mathrm{Hм}^{-2}, V_{0}=-2 \sqrt{a_{-2} a_{2}}$.

Соответствующие графики энергетических спектров в зависимости от главного и магнитного квантовых чисел и индукции магнитного поля представлены на рис. 2.

Заключение. В данной работе для частицы, движущейся в постоянном магнитном поле и испытывающей воздействие модельного потенциала квантового кольца (1), найдены аналитические выражения для энергий и волновых функций в терминах биконфлюэнтных функций Хойна, что позволяет расширить класс точно решаемых задач, возникающих при решении уравнения Шрёдингера.

Проведено сравнение полученных аналитических решений с результатами других авторов.

Предлагаемый модельный потенциал и соответствующие решения стационарного уравнения Шрёдингера могут быть использованы при описании процессов туннелирования в наносистемах [4], описываемых двухямными потенциалами.

Расчёты и построение графиков были выполнены с использованием систем символьной математики MathCAD 14 и Maple 14.

\section{БИБЛИОГРАФИЧЕСКИЙ СПИСОК}

1. А. Г. Уиверидзе, "Квазиточнорешаемые модели квантовой механики" // Физика элементарных частии, и атомного ядра, 1989. Т. 20, №5. С. 1185-1245; англ. пер.: A. G. Ushveridze Quasi-exactly solvable models in quantum mechanics // Soviet J. Particles and Nuclei, 1989. Vol. 20, no. 5. Pp. 504-528.

2. W.-C. Tan, J. C. Inkson, "Electron states in a two-dimensional ring - an exactly soluble model" // Semicond. Sci. Technol., 1996. Vol. 11, no. 11. Pp. 1635-1641.

3. H. A. Mavromatis, "Generalization of Casas-Plastino potentials to three dimensions" // Amer. J. Phys., 2000. Vol. 68, no. 3. Pp. 287-288.

4. А. А. Бръззгалов, Ф. И. Карманов, "Метод расщепления по физическим факторам в задаче о временной динамике волновых функций электронов двумерного квантового кольца" // Матем. моделирование, 2010. Т. 22, №6. С. 15-26; англ. пер.: A. A. Bryzgalov, F. I. Karmanov, "Method for splitting into physical processes in the problem on the time dynamics of electron wave functions of a two-dimensional quantum ring" // Math. Models Comput. Simul., 2011. Vol. 3, no. 1. Pp. 25-34.

5. S. Yu. Slavyanov, W. Lay, Special functions. A unified theory based on singularities.. New York: Oxford University Press, 2000. xvi+293 pp.; русск. пер.:С. Ю. Славянов, В. Лай, Специальные функции: Единая теория, основанная на анализе. СПб.: Невский Диалект, 2002. 312 c.

6. E. R. Arriola, J. S. Dehesa, A. Zarzo, "Spectral properties of the biconfluent Heun differential equation" // J. Comput. Appl. Math., 1991. Vol. 37, no. 1-3. Pp. 161-169.

7. А. Ф. Никифоров, В. Б. Уваров, Специальные функции математической физики. М.: Наука, 1984. 344 c. [A. F. Nikiforov, V. B. Uvarov, Special functions of mathematical physics. Moscow: Nauka, 1984. 344 pp.]

Поступила в редакцию $15 / \mathrm{XI} / 2012$;

в окончательном варианте - 27/I/2013. 
MSC: 81Q80, 81V65

THE ENERGY LEVELS AND EIGEN WAVE FUNCTIONS OF ELECTRONS IN QUANTUM RINGS IN THE MAGNETIC FIELD

\section{E. V. Antropova, A. A. Bryzgalov, F. I. Karmanov}

"National Research Nuclear University MPEI"

Obninsk State Technical University,

1, Studgorodok st., Obninsk, 249040, Russia.

E-mails: antrolen@yandex.ru, sandro185@mail.ru, fikarm@yandex.ru

The solution of the eigenvalue problem for non interacting electrons of the quantum ring in the magnetic field is discussed. The potential shape of the quantum ring permitting analytical solution was proposed. The solution of the appropriate eigenvalue problem was found in the terms of the Heun functions and expression for the energy levels was obtained. It was pointed out that proposed potential might be considered as a single-well or double-well potential of concentric quantum rings.

Key words: quantum ring, magnetic field, quasi-exactly solvable models of quantum mechanics.

Original article submitted 15/XI/2012;

revision submitted $27 / \mathrm{I} / 2013$.

Elena V. Antropova, Graduate Student, Dept. of General and Special Physics.

Aleksandr A. Bryzgalov, Postgraduate Student, Dept. of General and Special Physics.

Fedor I. Karmanov (Ph. D. (Phys. \& Math.)), Associate Professor, Dept. of General and Special Physics. 